Educación Física y Ciencia, vol. 19, n 1, e026, junio 2017. ISSN 2314-2561

Universidad Nacional de La Plata.

Facultad de Humanidades y Ciencias de la Educación.

Departamento de Educación Física

\title{
Componentes de control del entrenamiento utilizados por entrenadores escolares
}

\author{
Components of the training control used by school coaches
}

\section{Diego Alonso Alzate Salazar *; Carlos Federico Ayala Zuluaga *; Luis Gerardo Melo Betancourth *}

* Universidad de Caldas, Colombia | diego.alzate@ucaldas.edu.co; federico.ayala@ucaldas.edu.co; luis.melo@ucaldas.edu.co

\section{PALABRAS CLAVE \\ Pruebas de control}

\section{RESUMEN}

Con el propósito de determinar si los entrenadores que asistieron a los deportistas en los IV Juegos Deportivos Escolares Centroamericanos y del Caribe realizados en Armenia, Colombia 2013, aplicaron pruebas de control, fue desarrollado un estudio descriptivo y corte transversal donde fueron encuestados 41 entrenadores ( 36 de sexo masculino y 6 de femenino) de 13 modalidades deportivas y de 9 países entre los días 10 y 15 de noviembre de 2013. El promedio de edad de los entrenadores fue de 42,1 años $(11,8)$. Se suministró una encuesta que fue elaborada por el grupo investigador y la cual fue sometida a juicio de expertos. La información se trató en el programa SPSS (versión 20). Se encontró que 9 de cada diez entrenadores aplica pruebas de control del entrenamiento deportivo, siendo las más aplicadas las antropométricas con 28 respuestas, seguidas por las psicológicas y en última posición se encuentran las bioquímicas.

\section{KEYWORDS}

Control tests

Sportive training

Sportive education

\section{ABSTRACT}

Descriptive study and cross section were performed with the purpose of determining if coaches who attended the athletes in the IV Center-American and Caribbean sport scholar games performed in Armenia, Colombia in 2013 applied control tests in their practices. 41 coaches were surveyed (36 males, 6 females) of 13 sports and from 9 countries between November 10 and 15, 2013. The age average of the coaches was 42.1 years old (11. 8). A survey was prepared by the research team and it was submitted to experts' opinion. The information was analyzed through SPSS program (version 20). It was found that 9 out of every 10 coaches apply control tests of sport training, the anthropometric being the most applied one (28), followed by the psychological tests and in the last position the biochemical tests. 


\section{Introducción}

El control en el entrenamiento deportivo durante el proceso de formación y adaptación del deportista, es considerado como un componente de considerable importancia al momento de establecer el estado real de rendimiento del deportista (Montero, F. J. C., Peinado, P. J. B., Meléndez-Ortega, A., \& Gross, M. G., 2006; Borin, J. P., Prestes, J., Alfano M., \& Nélio., 2007; Serrato, 2008; Calderón, J., Benito, P., Meléndez, A., \& González, M., 2011; Cuadrado-Reyes, J., Chirosa, R.L., Chirosa, R.I., \& Aguilar-Martínez, D., 2012). Así mismo el control permite valorar objetivamente los componentes que se relacionan con su estado, además muchas veces, conocer a profundidad al deportista y ser sensible a los cambios que se pudieren presentar y así garantizar la consecución de los objetivos propuestos (Salado, J., Bazaco, M. J., Ortega, E., \& Gómez, M. Á. 2011; Alzate, S. D. A., Ayala, Z. C. F., \& Melo, B. L. G., 2015).

Además el entrenador puede identificar de forma subjetiva como se está presentando la evolución del deportista frente a las diferentes cargas propuestas, mediante la observación directa de este, así como la de valorarse propiamente a través de sus sensaciones (Faulkner, J., Parfitt, G., \& Eston, R., 2008; Liberal, R., \& García-Mas, A., 2011; Molinero, O., Salguero, A., \& Márquez, S., 2011).

En relación a lo establecido por el entrenador, de acuerdo a sus diferentes análisis objetivos y subjetivos, relacionados con la adquisición de habilidades, capacidades y del rendimiento esperado, éste entrará a hacer modificaciones al plan de entrenamiento, si se requiere, de tal forma que los objetivos propuestos se vayan alcanzando de forma paulatina (Borin et al., 2007; Corazza, S. T., Ribas, S. G., Santos, dos S. E., Rodrigues, da S. M., Piovesan, A. C., \& Alpes, A. C., 2015.; Yanci \& Los Arcos, 2013), es así como las diversas estrategias para realizar el control del entrenamiento deportivo, son de especial importancia para la eficacia de los procesos (Alzate, S. D. A., Ayala, Z. C. F., \& Melo, B. L. G., 2012; Alzate, et. al 2015).

Por tanto, el rol que desempeña el entrenador, además, debe ser enfocado a reunir un grupo de profesionales de diferentes disciplinas que coadyuven a la valoración y análisis de los resultados derivados del control desde esa misma interdisciplinariedad, mediante la aplicación de test válidos y precisos en las mediciones, sensibles a modificaciones, reproductibles en la obtención de valores idénticos o aproximados (Lopes, de F. D., Yukio, A. R., Pardono, E., Santos, dos S. R. J., Simões, H. G., \& Madrid, B., 2013; Thomas, J. R., Nelson, J. K., \& Silverman, S. J., 2007), de tal forma que no se conviertan en islas que no lleven a una convergencia en favor del alcance de los objetivos (Reilly, 2001; Viru \& Viru, 2003; Murcia, P. N., Taborda, CH. J., \& Ángel, Z. L. 2004; Weinek, 2005), y del adecuado desarrollo de los procesos formulados para el entrenamiento deportivo.

Por tanto, en el proceso de planificación del entrenamiento deportivo se hace necesario realizar pruebas iniciales (Faigenbaum, A.D., Loud, R.L., O'connell, J., Glover, S., O'connell, J., \& Westcott, W.L., 2001; Urdanpilleta, A., Martinez-Sanz, J., \& Lopez-Grueso, R., 2013.) para determinar en qué nivel se encuentran los deportistas y así elaborar un plan de trabajo, el cual debe ser evaluado periódicamente por parte del entrenador (Reilly, 2001; Viru \& Viru, 2003; Gómez 2004; Murcia et al, 2004; Weinek, 2005) y su equipo interdisciplinario, de tal manera que se monitoree dicho proceso y hacer ajustes si es necesario para lograr los objetivos inicialmente propuestos (Morales, 2005; Burgess \& Naughton, 2010; Pankhurst, A., Collins, D., \& Macnamara, Á., 2013) de acuerdo al calendario de competencias.

\section{Metodología}

Muestra: El estudio correspondió a un enfoque cuantitativo, estudio con alcance descriptivo y corte transversal, se encuestaron 41 entrenadores por conveniencia (35 hombres y 6 mujeres). Los entrenadores fueron abordados en los diferentes escenarios deportivos y espacios de descanso donde se les informó sobre el propósito de la investigación. Ya que la muestra no fue probabilística ni representativa, no se pretende 
hacer inferencia de los resultados.

Criterios de inclusión: entrenadores, directores técnicos o preparadores físicos inscritos oficialmente por sus respectivos países e incluidos en las delegaciones oficiales.

Procedimiento: Los datos fueron recolectados en la ciudad de Armenia -Colombia entre los días 10 y 15 de noviembre de 2013, en espacios como los escenarios de competencia como alojamiento y alimentación y de manera personal.

Instrumento: Se aplicó una encuesta que fue desarrollada en otro estudio con características similares por Álzate, et. al. (2012, 2015), además con base en la literatura existente en relación a la teoría y metodología del entrenamiento deportivo, la cual previamente fue diseñada por el grupo investigador basadas en bases de datos, análisis de diferentes bases de datos, experiencia de los investigadores y con el acompañamiento y asesoría de expertos en el área. Luego de ello fue sometida a validación por un grupo de académicos investigadores de varias Universidades colombianas, como por entrenadores con gran experticia en las temáticas relacionadas con los controles del entrenamiento deportivo, donde surgieron algunas recomendaciones, las cuales fueron tenidas en cuenta. La encuesta, fue aplicada personalmente por un grupo de colaboradores que fueron capacitados con anterioridad, y está constaba de 26 preguntas de las cuales 4 se relacionaron con el tema de control del entrenamiento deportivo. Las preguntas estaban relacionadas con:

\section{1. ¿Aplica pruebas de control? SI - NO}

2. ¿cuáles son las pruebas más utilizadas? A. antropométricas, B. bioquímicas, C. fisiológicas, D. laboratorio clínico, E. psicológicas, F. físicas

3. ¿cuántos son sus años de experiencia como entrenador?

4. ¿Cuál es su mayor nivel académico alcanzado? A. técnico, B. tecnológico, C. pregrado, D. posgrado.

Análisis y Estadística: El tratamiento de la información se hizo en el programa SPSS (versión 20), donde se utilizaron promedio, desviación típica y chi cuadrado para la significancia.

\section{Resultados y Discusión}

Características demográficas de la población: se encuestaron 41 entrenadores, 35 hombres (85,3\%) y 6 mujeres (34,7\%); se continua observando predominancia de la participación masculina en los procesos del desarrollo deportivo (Ayala, Z. C. F., Aguirre, L. H. H., \& Ramos, B. S., 2015; Robles, M. T. A., FuentesGuerra, F. J. G., Rodríguez, J. R., \& López, J. M. R., 2011). El promedio de edad fue del 42,1 años (11,8); el tiempo promedio como entrenador fue del 13,7 (8,7) y 9,2 años $(5,1)$ para hombres y mujeres respectivamente.

Al agrupar por nivel de estudios realizados, se encontró que el mayor porcentaje tiene estudios de formación profesional en los niveles de pregrado con 56,2\%; posgrado 26,8\%; técnico 9,7\% y tecnológico 7,3\%, lo encontrado se considera como óptimo, ya que más del $82 \%$ de los encuestados presenta estudios de formación profesional, lo cual es altamente satisfactorio para el contexto centroamericano, pues en otras investigaciones muchos de los entrenadores solamente poseen formación de pregrado (Ruiz \& Salinero, 2011; Robles et al., 2011), además la formación de posgrado subsidiará y apoyará de la mejor manera los procesos de desarrollo del deportista, desde la preparación inicial y en general (Bompa, 1983; Pérez, 2002).

$\mathrm{Al}$ momento de preguntar a los entrenadores si ellos aplican pruebas de control del entrenamiento deportivo, 
el 90,2\% respondió afirmativamente y el 9,8\% respondió de forma negativa, se encuentra como elemento preocupante los datos obtenidos a la pregunta de si se aplican pruebas de control, debido a la importancia que merece éste componente para alcanzar las metas propuestas (Morales, 2005; Pérez, 2008; Alzate et al. 2012; Alzate et al. 2015), así como también de "comprobar el nivel de preparación que el deportista obtiene en cada una de las etapas del proceso” (Gómez, 2002, s/p). El resultado anteriormente descrito guardo relación con Alzate, et al. (2012-2015) donde ambas investigaciones obtuvieron resultados similares ante la misma pregunta.

Según los resultados obtenidos se puede evidenciar que a pesar de la importancia de los procesos de control, aún existen entrenadores que hacen caso omiso de las diferentes recomendaciones y de los resultados de la literatura científica en relación a la justificación de la aplicación de dichos elementos (Murcia et al. 2004; Weinek, 2005; Pérez, 2008; Alzate et al. 2012-2015).

En la investigación realizada durante los Juegos Deportivos Escolares Centroamericanos y del Caribe, no se presentan diferencias significativas $(\mathrm{p}<0,05)$ en la aplicación de pruebas de control por género hay una correspondencia en los resultados, que evidencia que tanto los entrenadores masculinos y femeninos aplican pruebas de control indistintamente de su sexo. Lo anterior esta en relación con lo establecido por Alzate et al. (2015), donde la mayor parte de los entrenadores aplica pruebas de control independientemente del sexo.

Se encontraron diferencias altamente significativas entre la variable aplica pruebas de control y variable control antropométrico $(\mathrm{p}<0,002)$, control psicológico $(\mathrm{p}<0,006)$ y significativas con control fisiológico $(\mathrm{p}<0,017)$ y control físico $(\mathrm{p}<0,23)$.

Tipos de pruebas utilizadas: según la figura 1 (Pruebas de control utilizadas por los entrenadores), las pruebas de control que más utilizan los entrenadores son las antropométricas, seguidas de las psicológicas y fisiológicas, encontrándose que en cuarto lugar se encuentran las físicas, lo que genera sorpresa debido a que son las pruebas físicas especialmente las que pueden ser aplicadas directamente por el entrenador, aunque Menéndez (1984) y Pérez (2008), manifiestan que luego de los análisis preliminares se pueden plantear test tipo cualitativo (psicológicos) luego los cuantitativos, pero lo esencial es que respondan a los objetivos y propósitos planteados para el desarrollo del entrenamiento deportivo, lo anterior se diferencia de lo establecido por Alzate et al. (2012) y Alzate et al. (2015) donde ambas investigaciones arrojaron como resultado que las pruebas físicas son las más aplicadas por los entrenadores.

Figura 1: Pruebas de control utilizadas por los entrenadores

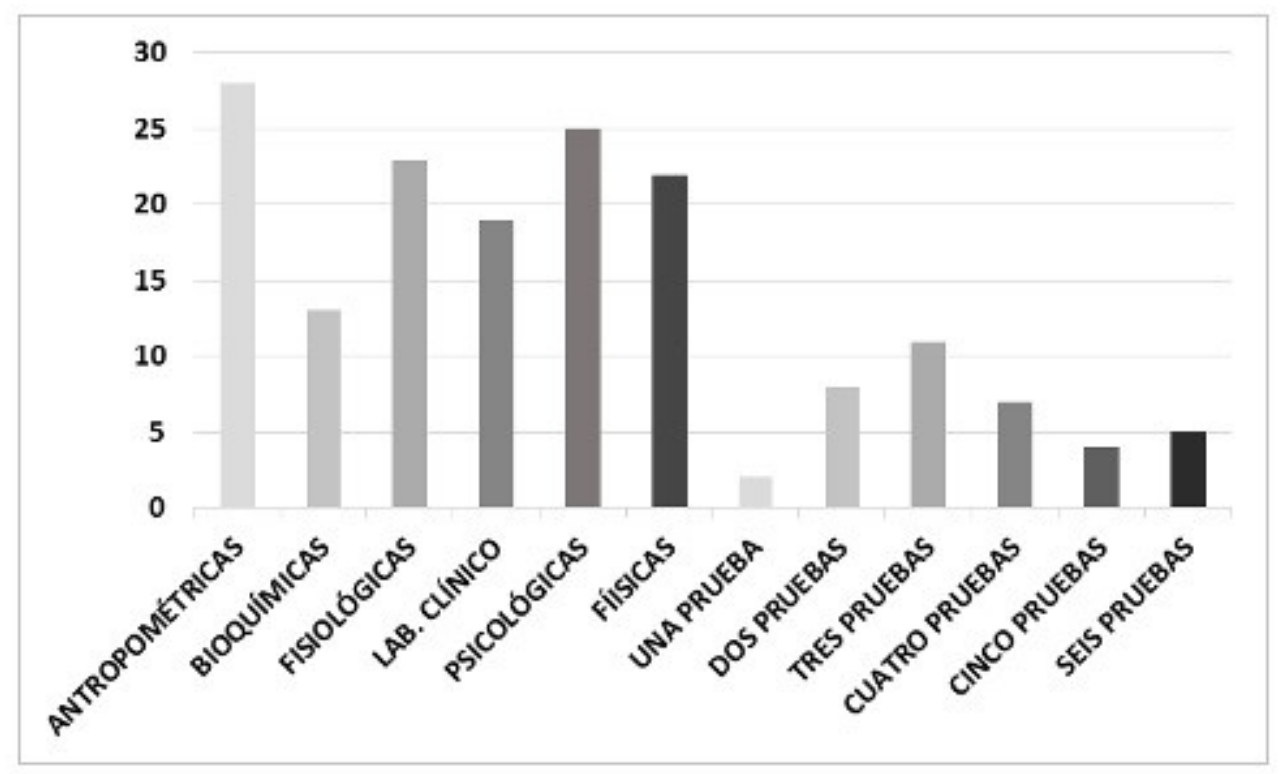


$\mathrm{Al}$ cruzar la variable tipo de deporte con pruebas utilizadas durante el proceso de entrenamiento deportivo, no se encontraron diferencias significativas $(\mathrm{p}<0,05)$ en ninguna de ellas, lo anterior no se relaciona con lo encontrado por Alzate et al. (2015) donde muestran que el grupo de deporte al que más se le aplica pruebas son a los de combate seguido de los de deportes de pelota.

Así mismo no se encontraron diferencias significativas $(\mathrm{p}<0,05)$ entre las variables años de experiencia como entrenador y aplica pruebas de control lo que indica que independiente de los años de experiencia que tenga como entrenador, este aplica pruebas de control.

\section{Conclusiones}

Consideramos que hay que insistir en hacer relaciones entre la formación y la experiencia de los entrenadores, para fortalecer los controles y evaluaciones, y así evitar posibles errores en los procesos de planificación y de formación deportiva.

Los aspectos anteriormente presentados, nos permiten entender que durante el proceso de entrenamiento los entrenadores de selección nacional solo realizan un papel de acompañamiento y asistencia táctica pues no existe el tiempo suficiente para la realización de las pruebas, además de los aspectos de contrataciones laborales definidas, lo que no garantiza continuidad en los procesos y afecta, limita e influye en la planificación del entrenamiento deportivo (Ramos, B. S., Ayala, Z. C. F., \& Aguirre, L. H. H, 2012), tal como lo muestran Alzate et al. (2015) la aplicación de pruebas y el respectivo seguimiento a estos deportistas se hacen durante su proceso de formación.

Agradecimentos: indicar de acordo com a carta de submissão

Agradecimientos: A los estudiantes de licenciatura en Educación Física y Deportes de la Universidad del Quindío, Armenia. Conflictos de interés: El manuscrito fue preparado y revisado con la participación de todos los autores, quienes declaramos que no existe ningún conflicto de intereses que ponga en riesgo la validez de los resultados presentados.

Financiamiento: Los autores declaramos que Vicerrectoría de Investigaciones y Posgrados (VIP) de la Universidad de Caldas, financio la investigación titulada "Caracterización de la metodología del entrenamiento deportivo, en los Juegos Intercolegiados Centroamericanos y del Caribe Armenia 2013” identificado con el código: VIP 1369514, y el presente trabajo proporcionando los rubros respectivos.

\section{Bibliografía}

Alzate. S. Diego, A., Ayala, Z., Carlos, F., Melo, B., \& Luis, G. (2012). Control del entrenamiento deportivo en juegos suramericanos Medellín 2010. Revista UDCA Actualidad \& Divulgación Científica, 15, 87-95.

Alzate, S., Diego, A., Ayala, Z., Carlos, F., Melo, B., \& Luis, G. (2015). Control del entrenamiento deportivo en deportistas escolares. Revista UDCA Actualidad \& Divulgación Científica, 18(2), 321-328.

Ayala-Zuluaga, C. F., Aguirre-Loaiza, H. H., \& Ramos-Bermúdez, S. (2015). Formación académica y experiencia deportiva de los entrenadores suramericanos. Revista Brasileira de Ciências do Esporte, 37(4), 367-375.

Bompa, T. O., \& Jones, D. (1983). Theory and methodology of training: The key to athletic performance. Dubuque, Iowa: Kendall/Hunt Pub. Co. 
Borin, J. P.; Prestes, J., Alfano M., \& Nélio. (2007b). Caracterização, Controle e Avaliação: Limitações e Possibilidades no Âmbito do Treinamento Desportivo. Rev. Treinamento Desportivo. (Portugal). 8(1):6-11.

Burgess, D. J.; \& Naughton, G. A. 2010. Talent development in adolescent team sports: a review. Int. J. Sports Physiol. Perform. (US). 5(1):103-116.

Corazza, S T; Ribas Smidt, G; dos Santos Soares, E; Rodrigues da Silva, M; Piovesan, A C; \& Alpes, A C; (2015). Análise do tempo de reação a partir do desempenho motor de adolescentes praticantes do nado Crawl. Motricidade, 11() 11-19. Recuperado de http://www.redalyc.org/articulo.oa?id=273043208003.

Faigenbaum, A. D.; Loud, R. L.; O'Connell, J.; Glover, S.; O'connell, J.; \& Westcott, W.L. 2001. The Effects of Different Resistance Training Protocols on Muscular Strength and Endurance Development in Children. J. Strength Cond. Res. (US). 5(4):459-65.

Faulkner, J., Parfitt, G. \& Eston, R. (2008). The rating of perceived exertion during competitive running scales with time. Psychophysiology, 45, 977-98.

Gomez, C. (2002). Control del proceso de entrenamiento deprotivo de alta competencia. Rev. Digital EFdeportes. (Argentina). Disponible desde internet en: http://:www.efdeportes.com/efd55/entren.html. (con acceso 4/08/2016). [links].

Liberal, R. \& García-Mas, A. (2011). Percepción de dolor y fatiga en relación con el estado de ánimo. Cuadernos de Psicología del Deporte, 11(2), 93-117.

Lopes de Farias, D; Yukio Asano, R; Pardono, E; dos Santos Silva, R J; Simões, H G; \& Madrid, B; (2013). Reprodutibilidade do teste anaeróbio de Wingate em ciclistas. Motricidade, 9 40-46. Recuperado de http://www.redalyc.org/articulo.oa?id=273029708006.

Menéndez, E. (1984). Planificación, control y análisis del entrenamiento deportivo en las categorías escolares. Cuba.

Molinero, O., Salguero, A. \& Márquez, S. (2011). Análisis de la recuperación-estrés en deportistas y relación con los estados de ánimo: Un estudio descriptivo. Cuadernos de Psicología del Deporte, 11(2), 47-55.)

Montero, F. J. C., Peinado, P. J. B., Meléndez-Ortega, A., \& Gross, M. G. (2006). Control biológico del entrenamiento de resistencia.(Biological control of endurance training). RICYDE. Revista Internacional de Ciencias del Deporte. 2(2), 65-87.doi: https://doig.org/10.5232/ricyde

Murcia, P.N.; Taborda, CH.J.; \& Angel, Z.L. 2004. Escuelas de formación deportiva y entrenamiento deportivo infantil. Kínesis (Colombia). 210p.

Pankhurst, A., Collins, D., \& Macnamara, Á. (2013). Talent development: linking the stakeholders to the process. Journal of sports sciences, 31(4), 370-380.

Pérez G, E. (2008). Las pruebas o tests en el deporte. Rev. Digital EFdeportes (Argentina). Disponible desde internet en: http://www. efdeportes. com/efd126/las-pruebas-o-tests-en-el-deporte. htm (con acceso 4/08/2016).[Links].

Pérez-Ramírez, Ma del Carmén. (2002). Caracterización del entrenador del alto rendimiento deportivo. Cuadernos de Psicología del Deporte; 2(1):15-37).

Ramos-Bermúdez, S., Ayala-Zuluaga, C. F., Aguirre-Loaiza, H. H. (2012). Planificación del Entrenamiento en Juegos Suramericanos Medellín 2010: Modelos, Duración y Criterios. Revista U.D.C.A. 15. pp. 67-76.

Reilly, T. 2001. Assessment of sports performance with particular reference to field games.Eur. J. Sport Science (Gran Bretaña). 1(3):1-12. 
Reyes, J. C., Ríos, L. J. C., Ríos, I. J. C., Tamayo, I. M., \& Martínez, D. A. (2012). La percepción subjetiva del esfuerzo para el control de la carga entrenamiento en una temporada en un equipo de balonmano. Revista de psicología del deporte, 21(2), 331-339. (con acceso 14/07/2015).

Robles, M. T. A., Fuentes-Guerra, F. J. G., Rodríguez, J. R., \& López, J. M. R. (2011). Perfil, experiencia y métodos de enseñanza de los entrenadores de jóvenes futbolistas en la provincia de Huelva. Retos: nuevas tendencias en educación física, deporte y recreación, (20), 21-25.

Ruiz, G., \& Salinero, J. J. (2011). El entrenador de alto nivel en triatlón: entorno próximo y cualidades fundamentales para el rendimiento. Rev Int Cienc Deporte, 23(7), 113-25.

Salado, J., Bazaco, M. J., Ortega, E. \& Gómez, M. Á. (2011). Opinión de los entrenadores sobre distribución de contenidos técnico-tácticos y pedagógicos en distintas categorías de baloncesto de formación. Cuadernos de Psicología del Deporte, 11(2), 51-62.

Roa, M. S. (2008). Medicina del deporte. Universidad del Rosario.

Thomas, J. R., Nelson, J. K., \& Silverman, S. J. (2007). Métodos de pesquisa em atividade física (5aed). Porto Alegre: Artmed.

Urdampilleta, A., Martínez-Sanz, J. M., \& Lopez-Grueso, R. (2013). Valoración bioquímica del entrenamiento: herramienta para el dietista-nutricionista deportivo. Revista Española de Nutrición Humana y Dietética, 17(2), 73-83.

Viru, A.; \& Viru, M. 2003. Análisis y Control del Rendimiento Deportivo. Editorial Paidotribo.

Weineck, J. 2005. Entrenamiento Total. Editorial Paidotribo.

Yanci Irigoyen, J., \& Los Arcos Larumbe, A. (2013). Evolución del rendimiento aeróbico y anaeróbico en futbolistas profesionales tras la pretemporada. (Aerobic and anaerobic performance variation in professional soccer players after preseason). CCD. Cultura_Ciencia_Deporte. 文化-科技-体育, 8(24), 207-215. doi: https://doig.org/10.12800/ccd (con acceso: 24/06/2015) 